\title{
ASSOCIAÇÃO DA SULFAMETOXAZOL AO TRIMETHOPRIM NO TRATAMENTO DA MALÄRIA POR PLASMODIUM FALCIPARUM
}

\section{Resultados obtidos em 40 casos da região Centro Oeste do Brasil}

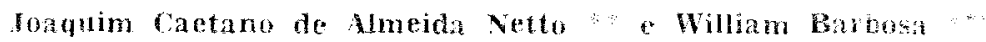

\begin{abstract}
A associacio da sulfametoxa:ol SHZ, ao Trimehoprim iTMP, jon ensalada em 40 portadores de malária pelo Plasmodium falciparum, en dua clapas $\mathrm{Na}$ primeira, foram observados 20 pacientes resistentes as 4-aminoquinoleinas. oligossintomáticos e com densidade parasitaria baixa. A administracio de 800 $m g$ de SMZ + $160 \mathrm{mg}$ de TMP a dez pacientes, em dose inica, bem como, didriamente durante 2 dirts, a 10 outros, se mostrou capaz de promover o desaparecimento dos trofozitos do sangue periférico em todos os 20 casos seis dentre 13 pacientes deste grupo apresentaram recrudeschncia da parasitemia assexuada durante o periodo de controle. de 30 dias. Não foram observadas ma. nifestaçóes de intolerância. Na segunda etapa foram obserrados mais $20 \mathrm{ma}$ cientes também. em sua maioria, resistentes as 4-aminaqunotenas, meninio agora casos com quadro clinico severo. A administraçáo de $1.600 \mathrm{mg} \mathrm{SHZ}+$ $320 \mathrm{mg}$ de TMP. diàriamente, durante 4 dias, promove a negativacão de parasitemia assexuada em todos os pacientes dentro de umi periodo de 82 a 96 horas. Dêste grupo, 2 aentre os 20 pacientes apresentaram recrudescencia cinata parasitária no pericdo de controle. Apenas 1 teve discreta anemia megaloolas. tica após o tratamento. que desapareceu espontâneamente.
\end{abstract}

\section{$\therefore T R O D U C A ̈ O$}

A ação antimalárica dos sulfamídicos $\therefore$ : descrita por Diaz de Leon (1937), que snseguiu cura de infecção por $P$. vivax - :lizando sulfamidicos 161. Coggeshall .938) publicou resultados de ensaio com = referidos compostos em malária de ma$\therefore$ cos e 3 anos após 11941 l em 30 casos de calária humana, demonstiando a eficácia - nnto de uma sulfona, o Promin, quanto a sulfadiazina sôbre os Plasmodium vivax. -aciparum e malariae 13 e 41 . Freire
Paraense i 944 , publicanem as wastitados de extenso e cuidadoso cibaio com a sul. fadiazina, sulfapiridina e sulfanilamida cm malária aviária (P. Gallindceum) consta. tando açăo terapeutica eficaz sobro os esquizontes tissulares e evitroctanos e falha sobre as formas sexuadas Neste ensaio a sulfadiazina se mostron sumenor as dois outros 110 , Findlay ools 1846 ! 11:blicaram os resultados do mpreos de tipos de sultamidicos en? 136 sasos de ma. lária pol $P$, falciparum em mopa ninopeis no Oeste da Átrica. tendo ovdenodado nemo

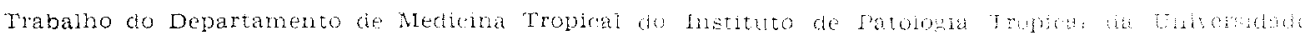
Federal de Guiás.

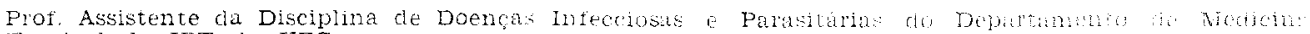
Tropical do IPT da UFGo.

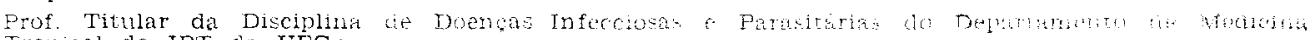
Iropical do IPT da UFGo

Ecoebio para publicacá esri $27 \cdot 9-71$
} 
esquizonticida sanguinea bastante inferior à das 4-aminoquinoleinas e do quinino, e ausência de ação sôbre os gametócitos 19 ! Greenberg (1949) demonstrou potenciali. zacáo da sulfadiazina pelo Proguanil con tra o P. gallinaceum 12 !. Rollo 1955 demonstrou que $1 / 8$ da dose minima eticaz (D.M.E.) de Pirimetamina $1 / 7$ D.ME de sulfadiazina, associadas, produziam o mesmo efeito da D.M.E. de cada uma das substancias quando empregadas isolada mente contra o $P$ gallinacenm 135 : Hurly (1959) demonstrou o mesmo tipo de potencialização, na ordem $1 / 10$ da D.M.E. df Pirmetamina ‥ $1 / 4$ da D.M.E. de sulfadiazina no tratamento de pacientes semi. imunes indigenas gambienses, infectados com $P$. falciparam $P$. malariate $F$. ovale (4).

Os estudos relacionados com a acáo an timalárica dos sulfamídicos despertaram contudo, até a decada de 1950. mais interêsse científico que prático. em lace da existência de esquizonticidas sanguineos potentes, baratos e de baixa toxicidade do grupo das 4 aminoquinolemas coloroquina e amodiaquinal. Dois fatos novos vieram. contudo, abrir perspectivas para o emprego prático dos conhecimentos em questão: de um lado o fenómeno da resistencia do P. falciparum às 4-aminoquinoleinas, doscrito, inicialmente, na Colombia por Moov e Lanier 1961), logo a seguir no Brasi? por Rodrigues da Silva e col. 1961: e no sudoeste da Ásia por Young e cols, ig63. Montegomery e cols 1963). Sandoshan cols. (1963), Powell e cols. 11963) e de outro lado o aparecimento das sultas de eliminacão lenta $(28,33,38.27,36$ e 31 ).

No Brasil, as observacóes iniciais de Rodrigues da Silva e col. sobre resistência do $P$. falcharum as 4-aminoquinoleinas. foram confirmadas posteriomente por vá rios outros pesquisadores. tanto em malaria experimental como em infeccōe naturais fRodrigues 1961, Galvão e cols 1961), Ferreira e col, 1963). Rodrigues da Silva t col. 1964). Schmidt. 1965 Barbosa e col. (1966). Neves e cols. 1968). Lopes e col $1966,1968+1969: 33,11$ 8. 34, 37, 1, 30, 20, 21 e 221.

Em decorrencia do exposto anterior. mente houve nos ultimos 8 anos, por parte dos malariologistas, grande interêsse pelo ensaio clínico da associação das sulfas de acão lenta e Pirimetamina. Atualmente ja se conta com um acêrvo apreciavel de dados sóbre a ação antimalárica desta associaçào que se tem mostrado un esquizonticida potente, eficaz mesmo contra as formas muli-resistentes di $P$. falciparum MeGregor e cols. 19631. De Gown ceols. 1964) Laing, 41964, 1966, 1966 \& 1969 . Lopes e col. (1966), Chin t cols. 1966:. Harinasuta e col., 1966), Verduager e cols.. . 1968) Netto e cols., 1968 , Walker e col.. 1968 : $126,5.16,17.18,19,20,2.13,36$. $39+401$

Tiès fatos, contudo. limitan atualment o mprêgo da associação sulta + pirimeta. mina como antimalárico

1 a grande tendencia que temi a Pirl. metamina de induzir ao fenomeno da le sisténcia, o que presumivelmente ammen. taria a possibilidade de vir a ocorrex resis tencia a esta associação.

2: a ocorencia ainda que pouco frequento de efeitos toxicos sôbre a crase sansuinea como leucopenia com neutropenia. agranulocitose, megaloblastose $\theta$ trombo. citopenia:

3) a nao disponibilidade da associa. cão sob a forma injetável, já que as formas mais graves se acompanham, quase sempre, de manilestacóes digestivas e neu rologicas que dificutam a adminjotracá de medicamentos por via oral.

Alom das himitacoes mencionadas oxist: a posibilidade anda que rara da overroncia de graves manifestacoes de intolorancia as sulfas como a sindrome de Ste. vens - Jonhson

Recentemente, un inibidor da redutase do ácido dihidrofólico pertencente ao grapo das diaminopirimidinas 10 Trimethoprim) ou seja o 2,4-diamino-5(3-4-5-trimetoxybenzyli pyrimidine, segundo observaçoes iniciais de Mantin e col. tem st mostrado menos tóxico que a Pirimetamina e revelado uma açáo antimaläica superior à daquela substancia acão que que se tem verificado mesmo em "rẹps" pirimetamino-resistentes l Martin : rol. (1967) i23)

Embora sejam ainda escassas as publi racōes sobre o emprêgo desta substancia no tratamento da malária humana, quet isoladamente, quer associada a sulfamidicos, os resultados iniciais têm sido animadores e a tolerancia nuito boa $(23,24$ 25): fato que nos levou a experimenta-la em infeceóes naturais por $P$. falciparum. 


\section{TERTAL: E METODOS}

Para eneaio clinteo da ascriaca suanetcxazol Tximethoprim ISMZ

‥P, i en malária observamos 2 on$\therefore$ distintus.

Grupo A: Iniciamos as observacoes en - ientes portadores de infeccão pelo Plas-

aum fatcipon am, recrudescentes de tra-

antos sinteriores con 4-aminoquinolei-

aprestntando quadro clinieo benigno.

$n$ estadio geral e olgossintomaticos.

... dersidade parasitaria baixa -... ate 10

... trotozotios por mm de sangue. Foram

wrados 10 pacentes en regine de in-

nacáo hospitnar e 10 ambulatorialmen-

Aos pacientes hospitalizados adminismos a compinido a dia de po - $580 / 9$ durance 2 dias e aos de ambulauma dose unica de 2 comprimidos.

- programado o contróle de cura duran30 dias através de hemoscopia de $12 / 12$ . as para os doontes internados. e de $3 / 3$ - s. para os doentes de ambulatorio. Fêzlém disso, contróe hematoiogico mesnte hemograma antes e apos o trata-nio, bem como mielograma apos o mesnos doentes internados, buscando sut -nace possiveis efeitos toxicos.

Grupo $E$ : Em face da boa tolerancia medicacalo obs rvada nos pacientos do 00 A. contiruamos o ensaio terapenda assoriaça SMZ. TMP en un srupo. meluindo agora pacientes em grande maioria com quadro clinico - declarado cmpregando um esquema de umps, 2 vêzes ao dia durante 4 dias secutivos para adultos o metade desta - durante u mesmo periodo para criancom menos de $30 \mathrm{~kg}$. A administrado medicamento for ijomosamente - rolada pela cifomagem. sendo que en acientes comatosos fol toita atraves de - ia nascóstrica. nos 3 primeiros dias ratamento Nos casos mais graves fol -ssario cuidadosa terapéutica de sustenD. oen cono correção dos distur. - hicireletrolíticos mediante cautelosa ...mistraçăo de solucōes balanceada.

- an coservados 2j pacientes segundo $\because$ esquema todos em regime de intemahospitalar. Para controle, além de -me clinico rooroso iepetido dian- mente. loi avelade mienimente nensi dade praslanda relacionada aos leucocitos mounte contagem de parasitas, em 100 campos meroscopicos de esfregaco de sangue periférico, coraco pelo Giemsa. Esta contagen toi repotida de $6 / 6$ horas durante todo o periodo de pateneia, e a seHuir de $12 / 2$ newas duante 30 dias. Alem deste contole, os pacienies foram subme. tidos aos joguntes exames laboratoriais Homognana completo por ocasiāo da intemacáo a segmir crolutivamente, mie-

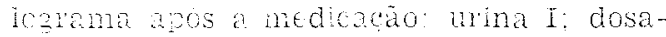
gens da neid da creatmina, do sodio, do petasio fas protonas sericas; deteminacous da bimtubina total e fracoes das transmmasos CO GF da fosfataso acaina: letwotes das proteinas do soro. Rx do toma o em algums, eletrocar ciograma

Neste grupo. \& pacientes an portadores de leinfecen a vegens de tratamento. 2 com recaide apos tratamento

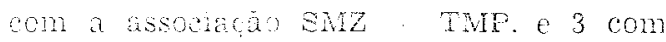
recaida apos thatarexto com t-aminoquinoleinas o 12 de primo infecea 12 virgens de tratanento 1 ratado com 4 -amimoduinoleinas sem exito, i com recaida apos matamento com quinino, 1 com recaida apos tratamento con associacáo SVZ - TMP e P portaderes de recaida apos o uso de 4-amincouinolemas: A densidade perastána antes do tratamento variou de 2 mil a 180 mil trofozoitos por mm de sansic. fol calouada muliplicando-se o nú moro do paratas compspondentes a 100 leucótes elo mmero de leucócitos do memograme divado por 100

\section{REOTIAOKS}

Consararam cil separacio os resulrades par a 1 a elupo fol "pioto" obscr. rado on a trablace de se hatear a dose itil cose media elicaz da associacóo medienmentese on estudo bem como de se conhecer os sens possiveis efeitos colate. rais. Conz se tratava de pacientes com estado geral relatianente bom oligossintomaticon. nos ouais a doenca na in picava em riscos inediatos pudemos enpoen: doses baxas da moducáa Ja no 
2. grupo constituido, em sua maioria de pacientes com manifestacōes clinicas importantes, em que $25 \%$ eram portadores de quadro clínico severo e em face da experiência que já tinhamos com a associacão medicamentosa em pauta. empregamos doso diaria mais elevada e a duracão do ras do inicio do tratamento. Apenas 13 pacientes 18 dentre os hospitalizados e 5 dentre os de ambulatóriol completaram o tempo de contrôle de cura previsto, ou seja, de 30 dias. Dêstes, 6 apresentaram lecrudescência da parasitemia entre o $99^{\circ}$ c $28^{\circ}$ dias do contrôle. Não foram obser-
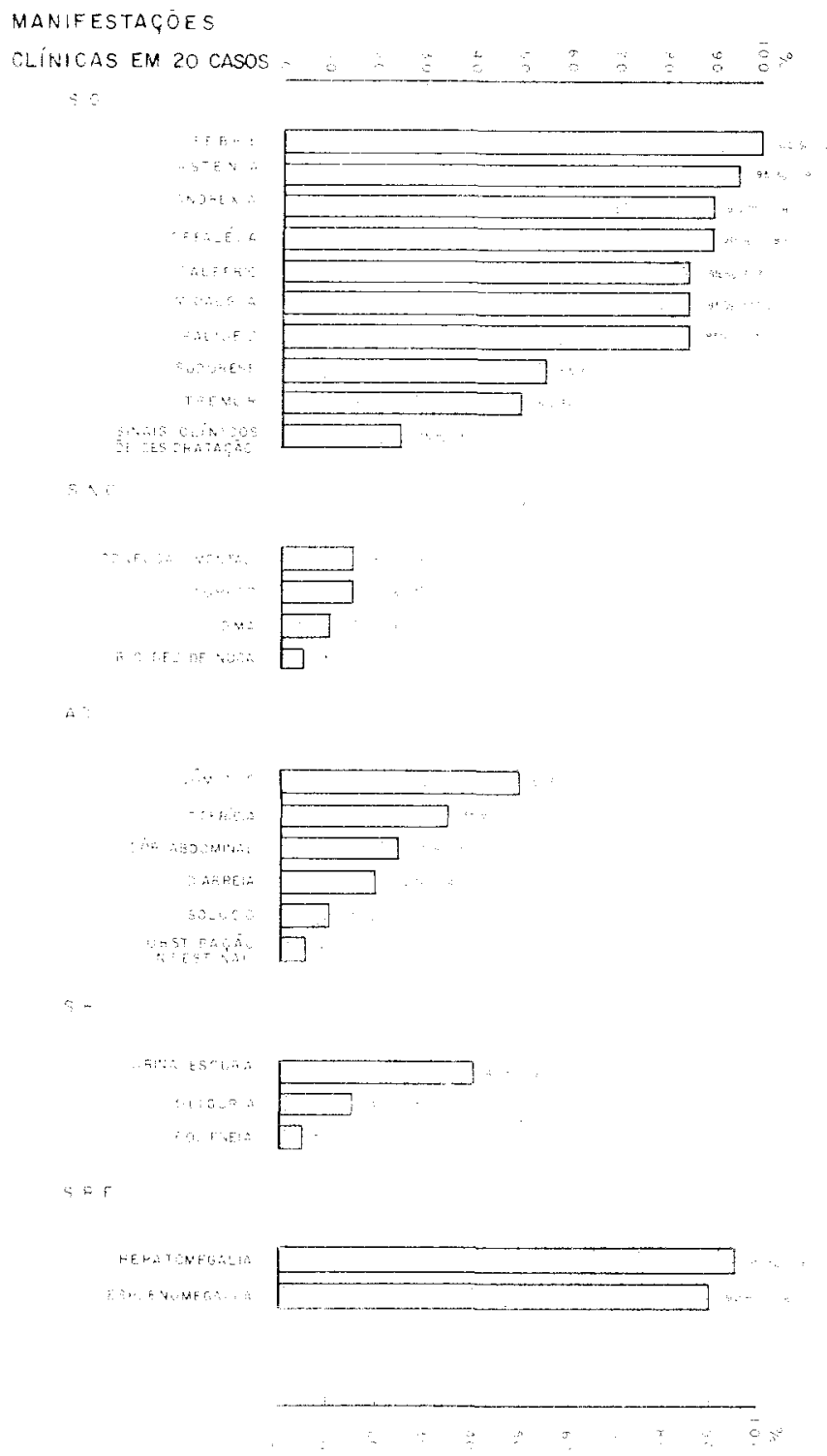

Fig. l

tratamento foi prolongada. Os dados clinicos do 2. grupo acham-se sumarizados na fig. 1

Grupo A: - En todos us 20 pacientes houve desaparecimento das formas sexuadas do sangue periférico entre 36 \& 72 ho- vadas manifestacōes clínicas de intolerância. e os hemogramas feitos em todos os pacientes, após o tratamento, bem como mielogramas em 4, nào apresentaram alteraçōes que pudessem ser atribuidas à medicação 
I-..po B: - Em todos os pacientes hou- .... nitida resposta parasitária nas pri$=-24$ horas. Todavia a resposta clí- $\quad \vdots$ se tornou bem evidente após o $20^{\circ}$ $\therefore \therefore$ : ratamento. O desaparecimento das ....... assexuadas do sangue periférico se :- - $:$ tre o $2 .^{\circ}$ e o $50^{\circ}$ dias, a maioria no - O desaparecimento da febre ocorreu $-\because 20^{\circ}$ e $060^{\circ}$ dias do início do trata$=\ldots$. sendo que $75 \%$ já se encontravam

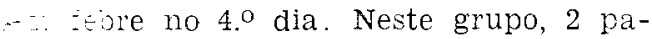
......5 apresentaram recrudescência clí- . - parasitária, um, no $160^{\circ}$ e outro no $\therefore$ Uias (fig. 2). A medicação parece dula óssea e os mielogramas, em nưmero de 8, mostraram, em 1 caso, alterações da série eritroblástica compativel com anemia tipo megaloblástico. Tôdas as outras alterações laboratoriais encontradas no início do tratamento desapareceram no decorrer do periodo de contrôle, exceto a hiperglobulinemia.

\section{DISCUSSÃO}

A análise dos nossos resultados mostra que a associação da Sulfametoxazol ao Trimethoprim na dose de $800 \mathrm{mg}$ de SMZ + $160 \mathrm{mg}$ de TMP em dose única, ou dià-

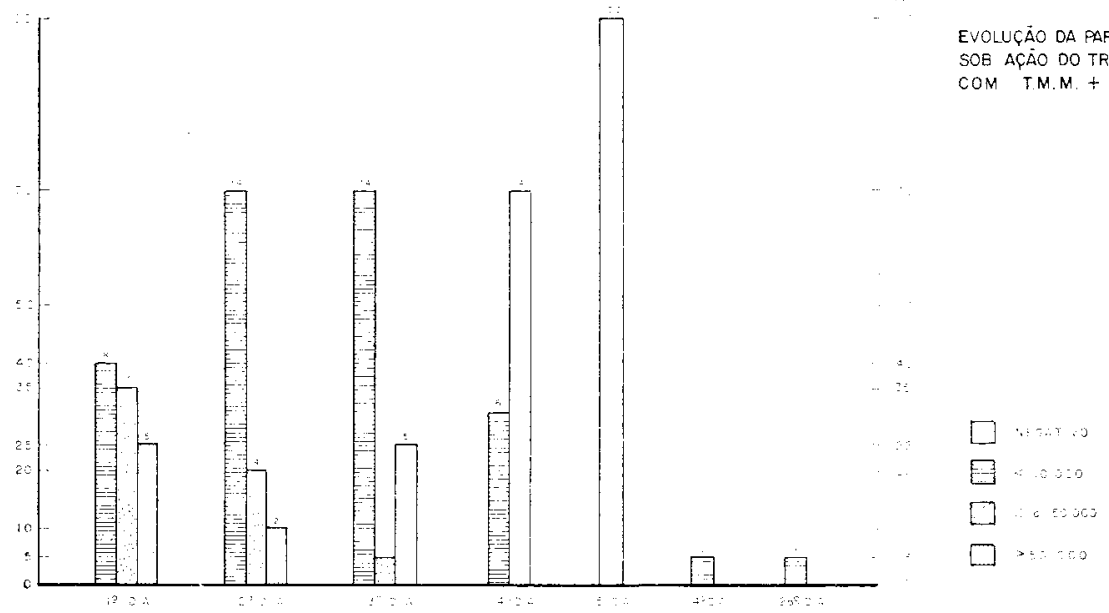

Fig. 2

E. ter interferido com a produção de ga$\because:=$ ócitos, pois todos os pacientes apresen:am formas sexuadas durante algum $\because m p o$, sendo que $50 \%$ durante todo o pe$\therefore$ do de contrôle e em densidade alta. -...iependentemente do indice parasitêmico $\therefore$.ive queda das taxas de hematias, do $\therefore$ matócrito e da hemoglcbina em todos :- casos, com tendência à normalização sservada na $3 .^{\mathrm{a}}$ ou $4 .^{\mathrm{a}}$ semana de con:-sie.

Quanto aos demais exames de labora:rio, 2 pacientes apresentaram cifras ele: $\vdots$ das de uréia e creatinina sendo que em $\ldots$ havia aumento concomitante de potás:3: 5 pacientes apresentaram aumento de :-irrubinas, 4 déles com parasitemia alta, - em 7 havia nítido aumento de globulizas.

Os hemogramas evolutivos não apre¿ntaram alteraçōes da série branca e das -iaquetas sugestivas de depressão da me- riamente durante 2 dias se mostrou capaz de promover a negativação das formas assexuadas em pacientes com baixa densidade parasitária e oligossintomáticos, presumivelmente semi-imunes, portadores de infecçōes por falciparum resistentes às 4aminoquinoleínas, dentro de um periodo relativamente curto. A parasitemia assexuada contudo veio a se tornar novamente patente em 6 dentre os 13 pacientes controlados durante 30 dias. Neste grupo preliminar a associação se mostrou bem tolerada e desprovida de efeitos colaterais nas doses empregadas, porém a percentagem de curas obtidas, segundo o critério de contrôle de 30 dias, foi muito baixa .

Considerando que as recaidas pelo $P$. falciparum podem ocorrer mais tardiamente, embora com menor freqüência, somos levados a admitir que a associação nas doses empregadas no $10^{\circ}$ grupo não 
curou mais do que $50 \%$ dos pacientes. Os res aos de Martin e Arnold (23) que conseguiram curar 6 voluntários não imunes inoculados com cêpas de $P$. falciparum não resistentes à cloroquina com $125 \mathrm{mg}$ de THM + $250 \mathrm{mg}$ de Sulfalene, em dose única.

Já no $2^{\circ}$ grupo, em que empregamos uma dose maior, $1600 \mathrm{mg}$ de SMZ - 320 de TMP ao dia, durante 4 dias, a incidência de recrudescimento foi muito menor durante o mesmo periodo de contrôle. Neste grupo, também predominaram as infecções resistentes à cloroquina, (11 dentre os 20 eram portadores de recrudescência clínica e parasitária, após tratamento com 4-aminoquinoleinas, 3 portadores de recrudescencia parasitária com o esquema anterior, 1 de recrudescência com o quinino e 5 virgens de tratamento). Os nossos resultados foram também aqui, inferiores aos de Martin e Arnold que, em um grupo de 11 voluntários não imunes inoculados com cêpa de $P$. falciparum resistente à cloroquina, conseguiram curar 10 dos 11 pacientes, com uma única dose de $500 \mathrm{mg}$ de Trimethoprim + $750 \mathrm{mg}$ de Sulfalene. Ressalte-se que no nosso $2 .^{\circ}$ grupo a dose total "per capita" foi de 1,29 g de $\mathrm{TMP} \div 6,4$ de $\mathrm{SMZ}$

Como neste grupo havia 5 casos graves com densidade parasitária alta, inclusive 2 complicados de coma, consideramos que a associação em estudo mostrou, segundo o esquema de 2 comps. ao dia durante 4 dias, uma potente ação esquizonticida, comparável, em nosso meio, à da cloroquina na dose total de $3,6 \mathrm{~g}$ em 4 dias para 0 adulto, e superior à da associação sulfametoxipirazina + pirimetamina na dose diária de $1,0 \mathrm{~g} \div 25 \mathrm{mg}$ respectivamente, durante 3 dias, segundo Netto e cols. (29).

Os resultados obtidos em nossa casuistica não foram tão animadores quanto os alcançados pelos autores já citados, porém nela foram incluidos pacientes com quadros clínicos graves, dado que a nosso ver torna significativos os resultados de nossa observação. Ressalte-se que o contrôle hematológico através de hemogramas semanais durante e após o tratamento, bem como de mielograma imediatamente após o tratamento em 12 casos, revelou alterações compativeis com anemia megaloblástica em apenas 1 caso (anemia do tipo macrocítica com hiperplasia do setor peritroblástico da medula óssea e presença de células megaloblastoides). Cumpre-nos, contudo, assinalar que em clinica particular observamos um paciente que após ingestão de 8 comprimidos, em 2 dias, apresentou púrpura trompocitopênica que se recuperou mediante suspensão do medicamento e terapêutica adequada.

Todavia esta alteração da hemostasia parece complicação rara, vez que não tem sido assinalada mesmo por autores que empregaram a associação SMZ - TMP por tempo prolongado. Drew e cols. (1967) a usaram durante um periodo de 3 meses na dose diária de $1.500 \mathrm{mg}$ de $\mathrm{SMZ}+$ $750 \mathrm{mg}$ de TMP, em um grupo de 10 pacientes, portadores de infecção brônquica crônica, incapacitante, como profilático. Observaram em apenas um caso plaquetopenia com prova do laço positiva, porém sem lesões purpúricas espontâneas (7). Kahn e cols. (1968) tratando 1 grupo de 14 pacientes portadores de infecção urinária crônica, durante um periodo que variou de 6 meses até mais de 1 ano, com dose diária de $4 \mathrm{~g}$ de SMZ $\nmid 1 \mathrm{~g}$ de TMP, observaram queda pronunciada das plaquetas em 1 caso somente (15).

O caso referido por nós com lesões purpúricas acompanhadas de intensa plaquetopenia em fase inicial da terapêutica parece ser o primeiro da literatura.

Neste caso a evolucão dos indices de plaquetas se comportou da seguinte maneira após a supressão de medicamento: de 12.000 por mm após a ingestão de 8 comprimidos em 2 dias, passou a 5.000 por $\mathrm{mm}^{3}$ no dia seguinte, sendo que a partir do $3 .^{\circ}$ dia da interrupção da terapêutica houve ascencão gradual das cifras de plaquetas que vieram a se normalizar no $17 .^{\circ}$ dia.

A tolerância à medicação nas doses empregadas na presente série foi boa, pois, com exceção das discretas perturbaçōes hematológicas encontradas em 1 caso, não foram observadas outras manifestaçóes de intolerância.

Consideramos que o RO 6-2580/9, no esquema de 4 comps./dia durante 4 dias, se revelou medicação eficaz para tratamento da malária pelo $P$. falciparum especialmente nos casos resistentes à cloroquina $\mathrm{e}$ à amodiaquina, tendo se mostrado de utilidade mesmo em casos graves e complicados dada sua rápida e potente ação ésquizonticida sanguínea. 


\section{SUMMARY}

The combination of a long acting sulfonamide, Sulfametoxazol, and a diiydrofolic acid redutase inhibitor, Trimethoprim, was employed in the treatment of 40 cases of natural infections falciparum malaria chloroquine resistant, ccording to two schedules.

On a first group of 20 patients with poor clinical manifestations and low Jarasitic average, sulfametoxazol $(0,800 \mathrm{gm})$ plus trimethoprim $(0,160 \mathrm{gm})$ were caministered in single dose to 10 patients and during two days, to 10 others. The clearance of asexual parasits occurred in all cases, however, 6 of 13 presented relupses during the cure control period of 30 days. No side clinical maniiestations were observed in this group.

On the second stage 20 cases most of them also chloroquine - resistant with severe clinical manifestations were treated with sulfametoxazol $(1.600 \mathrm{gm})$ juls trimethoprim $(0,320 \mathrm{gm})$ daily for four days. Clearence of trofozoits occurred in all cases during a time of 36 to 96 hours after therapy. Relapses occurred in 2 cases. Megaloblastic anemia occurred in 1 patient who recovered spontaneously.

\section{REFERÊNCIAS BIBLIOGRÁFICAS}

BARBOSA, W. E RODRIGUES DA SILVA. J. - Plasmodium falciparum resistentes às 4-aminoquinoleinas - Comunicação ao I Congresso Sudamericano - Del cono Sur, realizado em Buenos Aires, Agôsto de 1966. (Mimeografado).

- CHIN, W.; CONTACOS, P. G.; COATNEY, R. and KING, H. K. - The evaluations of sulfonamides, alone or in combination with Pyrimethamine in the treatment of multiresistant falci- parum malaria, Am. J. Trop. Med. Hyg., 15: 823, 1966.

COGGESHALL, L. T. - The cure of Plasmodium knowlesi malaria in Rhesus monkeys with sulfalamide and their susceptibility to reinfection. Amer. J. Trop. Med. 18: 715, 1938.

= COGGESSALL, L. T. and MAIER, J., - The effectiveness of two new types of chemotherapeutic agents in malaria. J. Am. M. Assoc. 117; 1077, 1941 .

三 DeGOWIN, R. L. and POWELL, R. D. - Drug-resistant falciparum malaria. J. Lab. Clin. Med. 64: 875, 1964.

¿ DIAZ DE LEÓN, A. - Primeiros casos de paludismo tratados por um derivado de la sulfanilamida. Bol. Ofic. Sanit. Pan-amer. 16: 1039, 1937.

- DREW, C. D. M.; HUGHES, D. T. D. and JENKINGS, G. C. - Long term treatment of chest infections with a combination of Thrimethoprim and Sulphonamide. The clinical, Bacteriological and hematological effects. Wien. Med. Akad. 5: 107, 1967.
8. FERREIRA, M. e ALMEIDA, D. Geographical distribuition of the strain of $P$. falciparum resistant to chloroquine WHO/MAC/917, 67, 1963.

9. FINDLAY, G. M.; MAEGRAITH, B. $G$. and col. - Investigations in the chemotherapy of malaria in west Africa. V. Sulphonamide compounds. Ann. Trop. Med. Paras. 40: 358 , 1946.

10. FREIRE, S. A and PARAENSE, W. L. - The prophylatic and curative action of sulfadiazine (2. sulfanilamide-Pyridine) and sulfanilamide ( $P$. amino - benzo - sulfanamide) on the erythro exoerithocytic cycles of Plasmodium gallinaceum (Therapeutic and parasitological aspects). Rev. Bras. Biol. 4: 27, 1944.

11. GALVÃO, A. L .; GUSMÃO, H. H. E.; SCHMIDT, A. W.; RICE, A. e MELLO, J. V. - Malária no Amapá Observações sôbre o comportamento anômalo do $P$. falciparum em face do tratamento pelas 4-aminoquiloneínas. Arch. Fac. Hyg. S. Publ. U. S. P. 15-16: 201, 1961, 1962 .

12. GREENBERG, J. - The potentiation of the antimalarial activity of Chlorguanide by p-aminobenzoic acid competitors, J. Pharmacol. 97: 238, 1949.

13. HARINASUTA, T. and VIRAVAN, C. Sulphormethoxine (Fanasil) and combinations of sulphormethoxine (Fanasil) and chloroquine or pyrimethamine (Daraprim) in the treatment of chloroquine resistant falciparum malaria in Thailand - Presented at the 11th Pacific Science Congress Tokyo, August, 1966. 
14. HURLY, M. G. D. - Potentiation of pyrimetamine by sulphadiazine in human malaria. Trans. Roy. Soc. Trop. Med. Hyg. 53: 412, 1959.

15. KAHN, S. B.; FREIN, S. A. and BRODSKY. - Effects of trimethoprim of folate metabolism in man. Clin. Pharm. Terap. 9: 550, 1958.

16. LAING, A. B. G. - Treatment of acute malaria with sulphorthomidine and a combination of sulforthomidine and pyrimethamine. Bull. WHO 34: 308,1966 .

17. LAING, A. B. G. - Antimalarial effects of sulformethoxine, Drapheny. sulfone and separate combinations of these with pyrimethamine: A review of preliminary investigations carried out in Tanzania, J. Trop. Med. Hyg. 71: 27, 1968a.

18. LAING, A. B. G. - Hospital and field trials of sulformethoxine with Pyrimethamine against Malaysian strains of Plasmodium falciparum and $P$. vivax. Med. J. Malaya. 23: 5, $1968 \mathrm{~b}$.

19. LAING, A. B. G. - Studies on the chemotherapy of malaria. I. The treatment of overt falciparum malaria with potentiation combinations of pyrimethamine and sulphormethoxine (sulfadoxine) or dapsone in the Gambia, Trans, Roy. Soc. Trop. Med. Hyg. 1969.

20. LOPES, P. F. A. e RODRIGUES DA SILVA, J. - Resistência do Plasmodium falciparum aos quimioterápicos. Apresentado ao Ir Congr. Soc. Bras. Med. Trop. Goiânia, Jan., 1966 .

21. LOPES, P. F. A. e SILVA, J. R. Chloroquine resistant, $P$. falciparum in South America. Proc. 8th Int. Cong. Trop. Med. Mal. 13941968.

22. LOPES, P. F. A.; RODRIGUES DA SILVA, J. e col. - Resistência do $P$. falciparum às 4-aminoquinoleínas. Estudo de 3 "raças" provenientes do Estado de Goiás. Rev. Soc. Bras. Med. Trop. 3: 73, 1969.

23. MARTIN, D. C. and ARNOLD, J. D. Trimethoprim and sulfalene in therapy of acute attacks of malaria. J. Clin. Pharm. 7: 336, 1967.

24. MARTIN, D. C. and ARNOLD, J. D. - Treatment of acute falciparum malaria with sulfalene and Trimethoprim. J.A.M.A. 203: 476, 1968.
25. MARTIN, D. C. and ARNOLD, J. D. - Trimethoprim and sulfanele Therapy of Plasmodium vivax. J. Clin. Pharmac. \& N.N. Drugs. 9: 155, 1969 .

26. MeGREGOR, I. A.; WILLIAMS, $K$. and GOODWIN, L. G. - Pyrimethamine and sulphadiazine in treatment of malaria. Brit. Med. J. 2: 728, 1963 .

27. MONTEGOMERY. R. and EYLIER, D. E. - Chloroquine rêsistant falciparum in Malaria. Trans. R. Soc. Trop. Med. Hyg. 57: 409, 1963.

28. MOORE, D. V. and LANIER, J. E. - Observation on two $P$. falciparum infections with an abnormal response to chloroquine. An. J. Trop. Med. and Hyg. 10: 5, 1961.

29. NETTO, J. C. A.; BARBOSA, W.; LOPES, P. F'. e RODRIGUES DA SILVA, J. - Associação Pirimetamina + Sulfametoxipirazina no tratamento da malária naturalmente adquirida. Resultados obtidos em 24 casos. - Trabalho apresentado no IV Congresso da Sociedade Brasileira de Medicina Tropical realizado em Recife, em fevereiro de 1968 - Resumo. Rev. Soc. Bras. Med Tropical. 2: 41, 1968

30. NEVES, J.; MAURA, H. B.; ARAÚJO, F. G.; COLEN, S. E. V. E LOYOLA, J. C. - Resistência do Plasmodium falciparum à cloroquina na Região do Paratú, no Estado de Minas Gerais. Rev. Inst. Med. Trop. São Paulo, 10: 28,1968 .

31. POWELL, R. D. BREWER, G. L. and ALVING, A. S. - Studies on a strain of chloroquine resistant $P$. falciparum from Vietnam, WHO/MAI/417, 1963.

32. RODRIGUES, D. C. - Casos de malária por Plasmodium falciparum resistentes ao tratamento pela clorocuina. Arq. Hyg. Saúde Pública, 26: 231,1961 .

33. RODRIGUES DA SILVA, J.; ALMEIDA LOPES, P. F.; MORTEO, R. e NAVEIRA, J. B. - Resistência do Plasmodium falciparum à ação da cloroquina, Hosp. 60: 581, 1961.

34. RODRIGUES DA SILVA, J. and LOPES, P F. A. - Cloroquine resistance in Plasmodium falciparum in Brazil. Rev. Bras. Med. Trop. 16: 301, 1964.

35. ROLLO, I. M. - The mode of action of sulphonamides, proguanil and Pyrimethamine on Plasmodium gallinaceum. Brit. J. Pharmacol. 10: 208, 1955 . 
$\because \quad$ SANDOSHAN, D. E.; EYLES, S. R.; MONTEGOMERY, R. - Drug resistance in Plasmodium falciparum malaria in South-East Asia. Med. J. Malaya, 18: 3, 1963.

:- SCHMIDT, S. - Fases evolutivas da 'uta antipalúdica na Regiăo Amazônica. Primeiros trabalhos realizados sobre resistência do $P$. falciparum às drogas cloroquinadas. Rev. Bras. Malariol. D. Trop. 17: 3, 1965.

$\because$ VERDRAGER, J; ROCHE, A. and CHOCEUM MUN CHEANG - Action de l'association diaphénylsulfone-p/ riméthamine sur le paludisme à Plas- modium falciparum au Camboge. (The combination of dapsone and $\mathrm{P} / \mathrm{rime-}$ thamine in treatment of Plasmodium falciparum malaria in Cambodia). Med. Trop. 28: 663, 1968.

39. YOUNG, J. D.; CONTACOS, P. G.; STITCHER, J. D. and MILLER, J. W. - Drug resistence in $P$. falciparum from Thailand. An. J. Trop. Med. and Hyg. 3: 305, 1963.

40. WALKER, A. J and ANTUÑANO LOPEZ, F. J. - Response to drugs of South American strains of Plasmodium falciparum. Trans. Roy Soc. Trop. Med. Hyg. 62: 654, 1968. 